\title{
Special Lifts of Ordinary K3 Surfaces and Applications*
}

\author{
Jeng-Daw Yu
}

\begin{abstract}
We study the cohomological properties of quasi-canonical lifts of an ordinary K3 surface over a finite field. As applications, we obtain a Torelli type theorem for ordinary K3 surfaces over finite fields and establish the Hodge conjecture for arbitrary self products of certain K3 surfaces over the field of complex numbers.
\end{abstract}

Keywords: K3 surfaces; canonical lifting.

\section{Introduction}

Let $X$ be an ordinary K3 surface over a finite field $k$. Then the deformation functor of $X$ admits a natural group structure ([N1, Theorem 1.6]). Under this group structure, the deformation of $X$ over an artinian local ring corresponding to the identity element is called the canonical lift of $X$. Deformations that correspond to torsion elements are called quasi-canonical lifts of $X$. In [N1], Nygaard uses these lifts to prove the Tate conjecture for ordinary K3 surfaces over finite fields.

In this paper, we investigate the cohomological properties of these canonical and quasi-canonical lifts of an ordinary K3 surface $X$ to a complete discrete valuation ring of characteristic zero. We show that these lifts are characterized

Received May 30, 2007.

2010 Mathematics Subject Classification.Primary 14J28; Secondary 14C25.

*This work was partially supported by Professor N. Yui's Discovery Grant from NSERC, Canada. 
by the existence of a (lifted) Frobenius acting on the Betti cohomology of the lifts. This property is similar to the well-known fact in the case of ordinary abelian varieties over a finite field. We also provide a Torelli type theorem for ordinary K3 surfaces over a finite field, which improves a result of Nygaard ([N2, Theorem 2.1]). On the other hand, we compute the Hodge group of the Hodge structure associated with a quasi-canonical lift of $X$. We then apply the result to establish the Hodge conjecture for arbitrary self products of certain K3 surfaces.

This paper consists of two parts and each part contains two sections. In Part I, $\S 1$, we investigate the basic properties of the quasi-canonical lifts of an ordinary K3 surface over a finite field, and in $\S 2$ we study the Hodge structures of these special lifts. Part II consists of some applications. More precisely, in $\S 3$, we prove a Torelli type theorem for ordinary K3 surfaces. In $\S 4$, we show that certain K3 surfaces over characteristic zero are quasi-canoncal lifts of their reductions. Using this fact, we translate the validity of the Tate conjecture for arbitrary self products of the ordinary reductions ([Z3]) to the validity of the Hodge conjecture via the explicit description of the Hodge groups of quasi-canonical lifts.

This paper contains the results of my thesis. I would like to thank my advisor, Professor Shing-Tung Yau, for his coherent support and encouragement. I would like to thank Professor Ching-Li Chai for the discussions of this work and answering my questions. Thanks are also due to Professor Richard Taylor for his interests in this work. The study of Hodge structures is also inspired by the discussions with Professor Noriko Yui and I would like to thank her heartily. Finally, I thank the referee for her/his careful reading and various suggestions leading to an improvement of the presentation.

\section{Notations}

Throughout this paper, we let $k$ be a perfect field of characteristic $p>0$ with countably many elements. Let $W=W(k)$ be the ring of Witt vectors of $k$ and $K=W \otimes_{\mathbb{Z}_{p}} \mathbb{Q}_{p}$. Let $\sigma: k \rightarrow k, W \rightarrow W, K \rightarrow K$ be the absolute Frobenius. We fix an algebraic closure $\bar{K}$ of $K$ with a fixed embedding $\bar{K} \subset \mathbb{C}$. For an $F$-(iso)crystal $H$ over $k$, denote by $\phi: H \rightarrow H$ the associated $\sigma$-linear Frobenius morphism. 


\section{Canonical and quasi-canonical lifts}

\section{Basic properties}

1.1. Let $X$ be an ordinary K3 surface over $k$. Let $\Phi$ be the formal Brauer group and let $\Psi$ be the enlarged formal Brauer group of $X$ ([AM, p.109 and Proposition IV.1.8]). Notice that $\Phi$ is of multiplicative type of height 1 and $\Psi=\Phi \oplus \Psi^{e t}$ is indeed a $p$-divisible group of height 21 . Here $\Phi$ is canonically identified with the connected component and $\Psi^{e t}$ is the maximal étale quotient of $\Psi$, respectively.

Let $\mathfrak{A n t}_{W}$ be the category of artinian local $W$-algebras. Let $\mathfrak{D e f} \mathfrak{f}_{X}$ be the deformation functor that sends an object $S$ in $\mathfrak{A n t}_{W}$ to the set of isomorphism classes of flat lifts of $X$ to $S$. We extend $\mathfrak{D} \mathfrak{e} \mathfrak{f}_{X}$ to formal lifts of $X$ over a complete local $W$-algebra $(R, \mathfrak{m})$ by setting

$$
\mathfrak{D e f} \mathfrak{f}_{X}(R):={\underset{n}{n}}_{\lim } \mathfrak{D e f} \mathfrak{f}_{X}\left(R / \mathfrak{m}^{n}\right)
$$

Similarly let $\mathfrak{D} \mathfrak{e} \mathfrak{f}_{\Psi}$ be the deformation functor of $\Psi$. Then $\mathfrak{D} \mathfrak{e} \mathfrak{f}_{\Psi}$ has a natural formal group structure. For a lift $\mathfrak{X}$ of $X$ to $S$, the associated enlarged formal Brauer group $\Psi_{\mathfrak{X}}$ of $\mathfrak{X}$ is a lift of $\Psi$ to $S$. Thus we get a natural transformation

$$
\gamma: \mathfrak{D e f} \mathfrak{f}_{X} \rightarrow \mathfrak{D e} \mathfrak{f}_{\Psi}
$$

It is known that $\gamma$ is an isomorphism of functors ([N1, Theorem 1.6]).

Definition 1.2. Let $S$ be an object in $\mathfrak{A r t}_{W}$ and $\mathfrak{X} \in \mathfrak{D e f}_{X}(S)$. The lift $\mathfrak{X}$ is called the canonical lift of $X$ to $S$ if the associated enlarged formal Brauer group $\Psi_{\mathfrak{X}}$ is the canonical lift of $\Psi$ to $S$ (i.e., $\Psi_{\mathfrak{X}}$ is the identity of the group $\mathfrak{D} \mathfrak{e} \mathfrak{f}_{\Psi}(S)$ ). The lift $\mathfrak{X}$ is called a quasi-canonical lift of $X$ to $S$ if $\Psi_{\mathfrak{X}}$ is a quasi-canonical lift of $\Psi$ (i.e., $\Psi_{\mathfrak{X}}$ is in the torsion subgroup of $\left.\mathfrak{D} \mathfrak{e} \mathfrak{f}_{\Psi}(S)\right)$ ([LST, $\left.\S 7\right]$ ). We also extend the definition to formal lifts over a complete local $W$-algebra.

1.3. Now let $R$ be a complete discrete valuation $W$-algebra with $[R: W]<\infty$. Let $L$ be the field of fractions of $R$. We fix a formal lift $\mathfrak{X}$ of $X$ to $R$. If $\mathfrak{X}$ is algebraizable, i.e. it is the formal completion of a (unique) scheme over $R$, we 
then use the same $\mathfrak{X}$ to indicate the scheme and set $X^{\circ}=\mathfrak{X} \otimes_{R} L$ to be the generic fiber of $\mathfrak{X}$.

Lemma 1.4. Let notations be as above. If $\mathfrak{X}$ is a quasi-canonical lift, then $\mathfrak{X}$ is algebraizable.

Proof. This is a direct generalization of [N1, Proposition 1.8]. For the reader's convenience, we include the proof here. We may assume that $k$ is the residue field of $R$. Let $\mathfrak{m}$ be the maximal ideal of $R$, and $R_{n}=R / \mathfrak{m}^{n}$. Write $\mathfrak{X}=\lim _{\longleftarrow} X_{n}$, where $X_{n}$ is a lift of $X=X_{0}$ to $R_{n}$ and the system $\left\{X_{n}\right\}$ defines $\mathfrak{X}$. By Grothendieck's existence theorem, it suffices to find an ample line bundle on $X$ that can be lifted to all $X_{n}$. Indeed, we will show that for any line bundle on $X$, a certain tensor power of it can be lifted to all $X_{n}$.

Let $\bar{k}$ be an algebraic closure of $k$ and $\bar{X}_{n}=X_{n} \otimes_{W} W(\bar{k})$. Consider the exact sequence of étale sheaves on $X_{n}$

$$
1 \rightarrow 1+\mathfrak{m} \mathcal{O}_{X_{n}} \rightarrow \mathcal{O}_{X_{n}}^{*} \rightarrow \mathcal{O}_{X}^{*} \rightarrow 1
$$

and the corresponding sequence on $\bar{X}_{n}$

$$
1 \rightarrow 1+\mathfrak{m} \mathcal{O}_{\bar{X}_{n}} \rightarrow \mathcal{O}_{\bar{X}_{n}}^{*} \rightarrow \mathcal{O}_{\bar{X}}^{*} \rightarrow 1
$$

We have a commutative diagram with injective vertical arrows

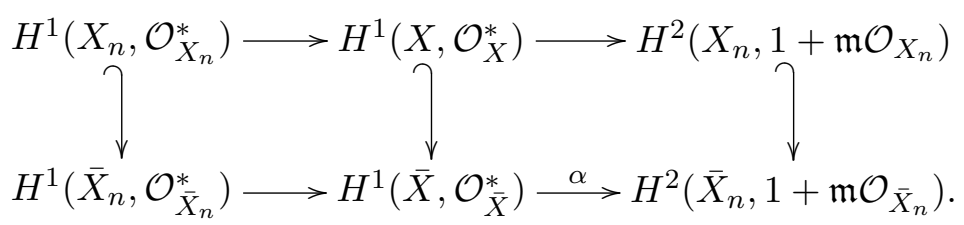

Thus it suffices to show that a certain multiple of the connecting map $\alpha$ vanishes. Henceforth we assume that $k$ is algebraically closed.

The map $\alpha$ factors through

$$
H^{1}\left(X, \mathcal{O}_{X}^{*}\right) \rightarrow \underset{r}{\lim } H^{1}\left(X, \mathcal{O}_{X}^{*} / \mathcal{O}_{X}^{*} p^{r}\right) \stackrel{\lim \beta_{r}}{\longleftrightarrow} H^{2}\left(X_{n}, 1+\mathfrak{m} \mathcal{O}_{X_{n}}\right)
$$

where $\beta_{r}$ is the connected morphism

$$
H^{1}\left(X, \mathcal{O}_{X}^{*} / \mathcal{O}_{X}^{*} p^{r}\right) \rightarrow H^{2}\left(X_{n}, 1+\mathfrak{m} \mathcal{O}_{X_{n}}\right)
$$


associated with the exact sequence of sheaves on $X_{n}$

$$
1 \rightarrow 1+\mathfrak{m} \mathcal{O}_{X_{n}} \rightarrow \mathcal{O}_{X_{n}}^{*} /\left(\mathcal{O}_{X_{n}}^{*}\right)^{p^{r}} \rightarrow \mathcal{O}_{X}^{*} /\left(\mathcal{O}_{X}^{*}\right)^{p^{r}} \rightarrow 1
$$

for $r \geq n$ (see [N1, p.218]). Let $\Psi_{n}$ be the enlarged formal Brauer group of $X_{n}$. There is a group isomorphism

$$
\delta: \mathfrak{D e f}_{\Psi}\left(R_{n}\right) \rightarrow \operatorname{Hom}_{\mathbb{Z}_{p}}\left(\lim _{\longleftarrow} H^{1}\left(X, \mathcal{O}_{X}^{*} / \mathcal{O}_{X}^{*} p^{r}\right), H^{2}\left(X, 1+\mathfrak{m} \mathcal{O}_{X_{n}}\right)\right)
$$

and furthermore we have $\lim _{\longleftarrow} \beta_{r}=\delta\left(\Psi_{n}\right)$ ([N1, Proposition 1.7]). Now by our assumption, the whole class $\lim _{\longleftarrow} \Psi_{n}$ is torsion. Hence a certain power of $\lim _{\longleftarrow} \beta_{r}$ vanishes.

We remark that if $p>2$, then one can apply the canonical coordinates of Deligne on the deformation space of $X$ (see [D2, §2.2], especially Théorème 2.2.2) to provide another proof of the assertion of lifting a line bundle.

Lemma 1.5. (Cf. [N1, Theorem 2.6].) With notations as in $\S 1.3$, the lift $\mathfrak{X}$ of $X$ is quasi-canincal if and only if $\mathfrak{X}$ is projective and the étale cohomology group $H_{\text {et }}^{2}\left(X_{\bar{K}}^{\circ}, \mathbb{Q}_{p}\right)$ attached to the generic fiber $X^{\circ}:=\mathfrak{X}_{L}$ splits

$$
H_{e t}^{2}\left(X_{\bar{K}}^{\circ}, \mathbb{Q}_{p}\right)=V_{0} \oplus V_{1} \oplus V_{2}
$$

such that

$$
V_{i} \otimes_{\mathbb{Q}_{p}} \mathbb{C}_{p} \simeq \mathbb{C}_{p}(-i)^{\oplus h_{i}}
$$

for $i=0,1,2$ as $\operatorname{Gal}(\bar{K} / L)$-modules. Here $\mathbb{C}_{p}$ is the completion of $\bar{K}$ and $(-i)$ denotes the Tate twist. (In this case, $h_{0}=h_{2}=1$ and $h_{1}=20$.)

Proof. Suppose $\mathfrak{X}$ is projective. Let $\Psi$ be the enlarged formal Brauer group of $X$ and $\mathfrak{G}$ be the enlarged formal Brauer group of $\mathfrak{X}$. Then the rational Tate module

$$
V(\mathfrak{G}):=\left(\lim _{\longleftarrow} \operatorname{Ker}\left\{p^{n}: \mathfrak{G}(\bar{K}) \rightarrow \mathfrak{G}(\bar{K})\right\}\right) \otimes_{\mathbb{Z}_{p}} \mathbb{Q}_{p}
$$

of $\mathfrak{G}$ is a $\operatorname{Gal}(\bar{K} / L)$-submodule of $H_{e t}^{2}\left(X_{\bar{K}}^{\circ}, \mathbb{Q}_{p}(1)\right)$ ([AM, Proposition IV.2.1 and Theorem IV.4.1]). Suppose that $H_{e t}^{2}\left(X_{\bar{K}}^{\circ}, \mathbb{Q}_{p}(1)\right)$ splits as in the Proposition. Then the $p$-divisible group $\mathfrak{G}$ is isogenous to the direct product $\Psi_{R}^{\circ} \times \Psi_{R}^{\mathrm{et}}$ over $R$. Here, $\Psi_{R}^{\circ}\left(\operatorname{resp} . \Psi_{R}^{\mathrm{et}}\right)$ denotes the unique lift of $\Psi^{\circ}$ (resp. $\left.\Psi^{\mathrm{et}}\right)$ to $R$. Thus $\mathfrak{G}$ 
is a quasi-canonical lift of its special fiber $\Psi([\mathrm{LST}, \S 7])$ and consequently $\mathfrak{X}$ is a quasi-canonical lift of $X$.

Conversely, if $\mathfrak{X}$ is quasi-canonical, it is projective by Lemma 1.4 and the $p$-adic étale cohomology group has the desired decomposition (see [N1, Theorem $2.6])$.

Lemma 1.6. With notations as in $\S 1.3$, a lift $\mathfrak{X}$ over $R$ of $X$ is quasi-canonical if and only if the Hodge filtration Fil ${ }^{\bullet}$ on $H_{d R}^{2}(\mathfrak{X} / R) \otimes_{R} L$ coincides with the one induced by the weight filtration on $H_{\text {cris }}^{2}(X / W)$ via the natural isomorphism

$$
H_{d R}^{2}(\mathfrak{X} / R) \otimes_{R} L \simeq H_{\text {cris }}^{2}(X / W) \otimes_{W} L
$$

([BO, Corollary 2.5]). In particular,

$$
\operatorname{Fil}^{2}\left(H_{d R}^{2}(\mathfrak{X} / R) \otimes_{R} L\right)=H^{0}\left(X, W \Omega^{2}\right) \otimes_{W} L .
$$

Proof. If $\mathrm{Fil}^{2}\left(H_{d R}^{2}(\mathfrak{X} / R) \otimes_{R} L\right)=H^{0}\left(X, W \Omega^{2}\right) \otimes_{W} L$, then the whole Hodge filtration $\mathrm{Fil}^{\bullet}$ on $H_{d R}^{2}(\mathfrak{X} / R) \otimes_{R} L$ coincides with that induced by the weight filtration on $H_{c r i s}^{2}(X / W)$ since the identification (1.6.1) is compatible with the cup products on both sides.

By definition, the lift $\mathfrak{X}$ is quasi-canonical if and only if the enlarged formal Brauer group $\Psi_{\mathfrak{X}}$ attached to $\mathfrak{X}$ is quasi-canonical. Recall that we have the commutative diagram ([NO, Theorem 3.20])

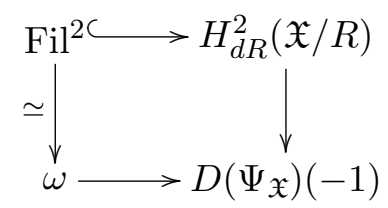

where $\omega$ and $D\left(\Psi_{\mathfrak{X}}\right)$ denote the space of invariant differential forms and the Dieudonné module of $\Psi_{\mathfrak{X}}$, respectively. Furthermore, the lift $\Psi_{\mathfrak{X}}$ is quasi-canonical if and only if

$$
\omega \otimes_{\mathbb{Z}_{p}} \mathbb{Q}_{p} \simeq H^{0}\left(X, W \Omega^{2}\right) \otimes_{W} L
$$

and the two vertical arrows of the above diagram are identified with projections (see loc. cit.). Consequently the assertion follows. 
Definition 1.7. Let notations be as in $§ 1.3$. Assume that $k$ is a finite field with $p^{a}$ elements and the lift $\mathfrak{X}$ is projective. We say that the Frobenius morphism on $X$ is liftable to $H^{2}(\mathfrak{X}(\mathbb{C}), \mathbb{Q})$ if there exists an endomorphism $\pi$ on $H^{2}(\mathfrak{X}(\mathbb{C}), \mathbb{Q})$ which satisfies the following two conditions:

(i) The induced endomorphism $\pi \otimes_{\mathbb{Q}} i d$ on $H^{2}(\mathfrak{X}(\mathbb{C}), \mathbb{Q}) \otimes_{\mathbb{Q}} \mathbb{Q}_{\ell}$ for every prime $\ell \neq p$ is the geometric Frobenius on $H_{e t}^{2}\left(X_{\bar{k}}, \mathbb{Q}_{\ell}\right)$ via the natural identification

$$
H^{2}\left(\mathfrak{X}(\mathbb{C}), \mathbb{Q}_{\ell}\right)=H_{\text {et }}^{2}\left(\mathfrak{X}_{\mathbb{C}}, \mathbb{Q}_{\ell}\right)=H_{\text {et }}^{2}\left(\mathfrak{X}_{\bar{K}}, \mathbb{Q}_{\ell}\right) \stackrel{\sim}{\longrightarrow} H_{\text {et }}^{2}\left(X_{\bar{k}}, \mathbb{Q}_{\ell}\right) .
$$

(ii) At the prime $p$, we have $\left(\pi \otimes_{\mathbb{Q}} i d\right) \otimes_{\mathbb{Q}_{p}} i d=\phi^{a} \otimes_{W} i d$ via the $p$-adic Hodge comparison $([\mathrm{F}, 6.1 .4])$

$$
\left(H^{2}(\mathfrak{X}(\mathbb{C}), \mathbb{Q}) \otimes_{\mathbb{Q}} \mathbb{Q}_{p}\right) \otimes_{\mathbb{Q}_{p}} \mathbf{B}_{\text {cris }}=H_{\text {et }}^{2}\left(\mathfrak{X}_{\bar{K}}, \mathbb{Q}_{p}\right) \otimes_{\mathbb{Q}_{p}} \mathbf{B}_{\text {cris }}=H_{\text {cris }}^{2}(X / W) \otimes_{W} \mathbf{B}_{\text {cris }} .
$$

(In particular, $\pi$ commutes with the Galois actions in both (i) and (ii).) In this case, we say that the Frobenius can be lifted to $H^{2}(\mathfrak{X}(\mathbb{C}), \mathbb{Q})$, and $\pi$ is called the lifted Frobenius.

Theorem 1.8. Let $\mathfrak{X}$ be a formal lift of an ordinary K3 surface $X$ over a finite field $k$ to a complete discrete valuation $W$-algebra $R$ with $[R: W]<\infty$. Then $\mathfrak{X}$ is quasi-canonical if and only if $\mathfrak{X}$ is projective and a power of the Frobenius morphism on $X$ is liftable to $H^{2}(\mathfrak{X}(\mathbb{C}), \mathbb{Q})$.

Proof. We may assume that $k$ is the residue field of $R$. Suppose $\mathfrak{X}$ is a quasicanonical lift. Let $X^{\circ}$ be the generic fiber of $\mathfrak{X}$ over the field of fractions $L$ of $R$. Then after replacing $k, R$ and $L$ by compatible finite extensions, there exist an abelian variety $A^{\circ}$ over $L$ with good reduction $\mathfrak{A}$ over $R$ and a $\mathbb{Z}$-algebra $C \subset \operatorname{End}_{L} A^{\circ}$ such that there exists an isomorphism of rational Hodge structures

$$
u: C^{+} P^{2}\left(X^{\circ}(\mathbb{C}), \mathbb{Q}(1)\right) \stackrel{\sim}{\longrightarrow} \operatorname{End}_{C} H^{1}\left(A^{\circ}(\mathbb{C}), \mathbb{Q}\right) ;
$$

furthermore, after extending the scalars to $\mathbb{Q}_{\ell}$ for every prime $\ell$, the map $u$ induces an isomorphism of $\operatorname{Gal}(\bar{K} / L)$-modules

$$
u_{l}: C^{+} P_{e t}^{2}\left(X_{\bar{K}}^{\circ}, \mathbb{Q}_{\ell}(1)\right) \stackrel{\sim}{\rightarrow} \operatorname{End}_{C} H_{e t}^{1}\left(A_{\bar{K}}^{\circ}, \mathbb{Q}_{\ell}\right)
$$


([D1, Proposition 6.5 and $\S 6.6]$ ). Here $P_{\bullet}^{2}(\bullet)$ is the primitive part in the corresponding cohomology group with respect to a chosen polarization and $C^{+}$denotes the even Clifford algebra. Let $A$ over $k$ be the special fiber of $\mathfrak{A}$. Then $A$ is an ordinary abelian variety and $\mathfrak{A}$ is isogenous to the canonical lift of $A$ ([N1, Proposition 2.5 and Corollary 2.8]). Let $\pi_{A} \in\left(\operatorname{End}_{R} \mathfrak{A}\right) \otimes_{\mathbb{Z}} \mathbb{Q}$ that lifts the Frobenius morphism of $A$. Via $u$, the map $\pi_{A}$ induces an endomorphism $\pi$ on $H^{2}\left(X^{\circ}(\mathbb{C}), \mathbb{Q}\right)$ which satisfies (1.7.1) (cf. [N1, Lemma 3.2]).

At the prime $p$, by applying $u_{p}$, tensoring with $\mathbf{B}_{\text {cris }}$ over $\mathbb{Q}_{p}$ and taking the $\operatorname{Gal}(\bar{K} / L)$-invariants, we get an isomrphism of $F$-isocrystals

$$
u_{\text {cris }}: C^{+}\left(P_{\text {cris }}^{2}(X / W) \otimes_{W} K(1)\right) \stackrel{\sim}{\rightarrow} \operatorname{End}_{C}\left(H_{\text {cris }}^{1}(A / W) \otimes_{W} K\right)
$$

([F, Théorème 6.1.4]). Since $\pi_{A}$ is the lifted Frobenius morphism on $A$, the induced map of $\pi_{A}$ on $H_{c r i s}^{1}(A / W) \otimes K$ is just the geometric Frobenius $\phi^{a}$. Here $\phi$ is the absolute Frobenius on the crystalline cohomology and $p^{a}$ is the cardinality of $k$. Then it is easy to see that the induced map of $\pi$ on $H_{c r i s}^{2}(X / W)$ is the geometric Frobenius morphism since $u_{c r i s}$ transfers the absolute Frobenius to the absolute Frobenius.

Conversely, by a base change of $k$, we assume that the Frobenius endomorphism of $X$ over $k$ can be lifted to $\pi$ on $H^{2}\left(X^{\circ}(\mathbb{C}), \mathbb{Q}\right)$. Suppose the field $k$ has $p^{a}$ elements. Since $X$ is ordinary, the characteristic polynomial $f_{\phi}(x)$ of the absolute Frobenius $\phi$ on $H_{c r i s}^{2}(X / W)$ decomposes as $f_{\phi}(x)=f_{\phi}^{(0)}(x) f_{\phi}^{(1)}(x) f_{\phi}^{(2)}(x)$ according to the valuation of its roots. (Although $f_{\phi}(x)$ is not well-defined, the valuations of its roots, the $a$-th powers of the roots, and the decomposition of the crystal $H_{c r i s}^{2}(X / W)$ are well-defined.) Now the roots of the characteristic polynomial $f_{\pi}(x)$ on $H^{2}\left(X^{\circ}(\mathbb{C}), \mathbb{Q}\right)$ are $a$-th powers of the roots of $f_{\phi}(x)$. Thus $f_{\pi}(x)$ decomposes as $f_{\pi}(x)=f_{\pi}^{(0)}(x) f_{\pi}^{(1)}(x) f_{\pi}^{(2)}(x) \bmod p$. By the Gauss Lemma, $f_{\pi}(x)$ has a decomposition over $\mathbb{Z}_{p}$ that lifts the decomposition $\bmod p$. Thus $H_{e t}^{2}\left(X_{\bar{K}}^{\circ}, \mathbb{Q}_{p}\right)$ has a decomposition as in Lemma 1.5 and consequently $\mathfrak{X}$ is a quasi-canonical lift.

\section{The Hodge structure}

2.1. Let notations be as in $\S 1.3$. In the sequel, we further suppose that $k$ is a finite field and the lift $\mathfrak{X}$ of the ordinary $\mathrm{K} 3$ surface $X$ is quasi-canonical. Recall 
that $X^{\circ}$ is the generic fiber of $\mathfrak{X}$. Then Theorem 1.8 says that there exists an endomorphism " $\pi^{n}$ " on $H^{2}\left(X^{\circ}(\mathbb{C}), \mathbb{Q}\right)$ which lifts the $n$-th iteration of the Frobenius endomorphism of $X$ relative to $k$ for some positive integer $n$.

Lemma 2.2. Let notations be as above. With respect to the embedding $L \subset$ $\bar{K} \subset \mathbb{C}$, the Hodge structure $H^{2}\left(X^{\circ}(\mathbb{C}), \mathbb{Q}\right)$ of the complex projective K3 surface $X^{\circ} \otimes_{L} \mathbb{C}$ is determined by the lifted Frobenius $\pi^{n}$ and the map $\pi^{n}$ is a Hodge cycle.

Proof. We need to figure out the 1-dimensional sub- $\mathbb{C}$-vector space

$$
H^{0}\left(X_{\mathbb{C}}^{\circ}, \Omega_{X_{\mathbb{C}}}^{2}\right)=\operatorname{Fil}^{1}\left(H_{d R}^{2}\left(X_{\mathbb{C}}^{\circ} / \mathbb{C}\right)(1)\right) \subset H_{d R}^{2}\left(X_{\mathbb{C}}^{\circ} / \mathbb{C}\right)(1)=H^{2}\left(X^{\circ}(\mathbb{C}), \mathbb{C}\right)(1) .
$$

We consider $\overline{\mathbb{Q}}$ as a subfield of $\mathbb{C}$. Thus the embedding $\bar{K} \subset \mathbb{C}$ provides an embedding $\overline{\mathbb{Q}} \subset \bar{K}$ and $\overline{\mathbb{Q}}$ is equipped with the induced $p$-adic valuation.

Let $H=H^{2}\left(X^{\circ}(\mathbb{C}), \mathbb{Q}(1)\right)$ and let

$$
\begin{aligned}
H_{>0} & =\left(H^{0}\left(X, W \Omega^{2}\right) \otimes_{W} K(1)\right) \otimes_{K} \mathbb{C} \\
H_{0} & =\left(H^{1}\left(X, W \Omega^{1}\right) \otimes_{W} K(1)\right) \otimes_{K} \mathbb{C} \\
H_{<0} & =\left(H^{2}(X, W \mathcal{O}) \otimes_{W} K(1)\right) \otimes_{K} \mathbb{C} .
\end{aligned}
$$

Then on $H_{\mathbb{C}}=H_{d R}^{2}\left(X^{\circ} / L\right)(1) \otimes_{L} \mathbb{C}$, we have

$$
H_{\mathbb{C}}=H_{>0} \oplus H_{0} \oplus H_{<0} .
$$

Since $\mathfrak{X}$ is a quasi-canonical lift, the filtration is given by Fil ${ }^{1} H_{\mathbb{C}}=H_{>0}$, which is 1-dimensional over $\mathbb{C}$, and $\pi$ acts on it through the first factor $H^{0}\left(X, W \Omega^{2}\right) \otimes_{W}$ $K(1)$ of $H_{>0}$ as multiplication by a constant $q \epsilon$, where $q$ is an integer power of $p$ and $\epsilon$ is a $p$-adic unit. Thus Fil $^{1} H_{\mathbb{C}}$ is the unique complex 1-dimensional eigenspace of $\pi$ in $H_{\mathbb{C}}$ attached to the unique eigenvalue with positive $p$-adic valuation.

Lemma 2.3. With the notations as in $\S 2.1$, the Frobenius morphism on $X$ relative to $k$ can be lifted to an endomorphism $\pi$ on $H^{2}\left(X^{\circ}(\mathbb{C}), \mathbb{Q}\right)$ and the map $\pi$ is a Hodge cycle.

Proof. We will abuse notations to let $\pi$ be the geometric Frobenius on any cohomology of $X$. Take a positive integer $n$ such that the iterated Frobenius $\pi^{n}$ can 
be lifted to $H^{2}\left(X^{\circ}(\mathbb{C}), \mathbb{Q}\right)$, still call it $\pi^{n}$, and such that $\pi^{n}=p^{a}$ on the algebraic cycle classes in $H^{2}\left(X^{\circ}(\mathbb{C}), \mathbb{Q}\right)$ for a suitable integer $a$. By Lemma 2.2, we can regard $\pi^{n}$ as a class $\left[\pi^{n}\right]$ in

$$
\text { End } H^{2}\left(X^{\circ}(\mathbb{C}), \mathbb{Q}\right) \subset H^{4}\left(X^{\circ}(\mathbb{C}) \times X^{\circ}(\mathbb{C}), \mathbb{Q}(2)\right),
$$

where End $H^{2}\left(X^{\circ}(\mathbb{C}), \mathbb{Q}\right)$ is the algebra of endomorphisms of the rational Hodge structure $H^{2}\left(X^{\circ}(\mathbb{C}), \mathbb{Q}\right)$.

Pick a prime $\ell \neq p$. Then the class $\left[\pi^{n}\right]$ in $H^{4}\left(X^{\circ}(\mathbb{C}) \times X^{\circ}(\mathbb{C}), \mathbb{Q}_{\ell}(2)\right)=$ $H_{e t}^{4}\left(X_{\bar{k}} \times X_{\bar{k}}, \mathbb{Q}_{\ell}(2)\right)$ is an algebraic cycle class of $X_{\bar{k}} \times X_{\bar{k}}$ since it is represented by the graph $\Gamma_{\pi^{n}}$ of the endomorphism $\pi^{n}$ on $X$. We know that the set of algebraic cycle classes (modulo homological equivalence with $\mathbb{Q}$ coefficients $) C^{2}\left(X_{\bar{k}} \times X_{\bar{k}}, \mathbb{Q}\right) \subset H_{e t}^{4}\left(X_{\bar{k}} \times X_{\bar{k}}, \mathbb{Q}_{\ell}(2)\right)$ are $\mathbb{Q}$-spanned by $\operatorname{pr}_{i}^{*}[X]$, $\operatorname{NS}\left(X_{\bar{k}}\right) \times \operatorname{NS}\left(X_{\bar{k}}\right)$ and the graphs of the iterations of $\pi^{n}$ ([Z2, Corollary 6.1.1]). Here $p r_{i}$ are the projections $X \times X \rightarrow X$ to the $i$-th factor for $i=1,2$. Notice that in this case, $\mathrm{NS}\left(X_{\mathbb{C}}^{\circ}\right)_{\mathbb{Q}} \rightarrow \mathrm{NS}\left(X_{\bar{k}}\right)_{\mathbb{Q}}$ is an isomorphism ([N1, Theorem 3.3]). Thus the class $[\pi] \in C^{2}\left(X_{\bar{k}} \times X_{\bar{k}}, \mathbb{Q}\right)$ can be obtained as a $\mathbb{Q}$-combination of $\left[\pi^{n}\right],\left[\pi^{2 n}\right]=\left[\pi^{n}\right] \circ\left[\pi^{n}\right], \cdots$ and elements in $\operatorname{NS}\left(X_{\mathbb{C}}^{\circ}\right) \times \operatorname{NS}\left(X_{\mathbb{C}}^{\circ}\right)$. Now, via (2.3.1), $\mathrm{NS}\left(X_{\mathbb{C}}^{\circ}\right)_{\mathbb{Q}} \times \mathrm{NS}\left(X_{\mathbb{C}}^{\circ}\right)_{\mathbb{Q}}$ is a subspace of End $H^{2}\left(X^{\circ}(\mathbb{C}), \mathbb{Q}\right)$. Consequently $[\pi]$ can be regarded as an element in $\operatorname{End} H^{2}\left(X^{\circ}(\mathbb{C}), \mathbb{Q}\right)$. The assertions now follow.

2.4. Now let $Z$ be a K3 surface over the field $\mathbb{C}$ of complex numbers. Let $\operatorname{NS}(Z)^{\perp}$ be the orthogonal complement of the Néron-Severi group $\operatorname{NS}(Z)$ in $H^{2}(Z, \mathbb{Z}(1))$ with respect to the cup product pairing. Then $\mathrm{NS}(Z)^{\perp}$ is a sub-integral Hodge structure of $H^{2}(Z, \mathbb{Z}(1))$. Let $\mathrm{M}(Z)=\mathrm{NS}(Z)^{\perp} \otimes_{\mathbb{Z}} \mathbb{Q}$.

Let $\mathbb{S}=\operatorname{Res}_{\mathbb{C} / \mathbb{R}} \mathbb{G}_{m}$ be the Deligne torus and

$$
h: \mathbb{S} \rightarrow \mathrm{GL}\left(\mathrm{M}(Z)_{\mathbb{R}}\right)
$$

be the associated homomorphism that induces the Hodge structure on $\mathrm{M}(Z)$. Let $\mathrm{Hdg}_{Z}$ be the Hodge group of $h$ and $\mathrm{E}=\operatorname{End}_{\mathrm{Hdg}_{Z}} \mathrm{M}(Z)$. Then $\mathrm{E}$ is a finite field extension of $\mathbb{Q}$. Thus $\mathrm{M}(Z)$ is a finite dimensional vector space over $\mathrm{E}$ with an

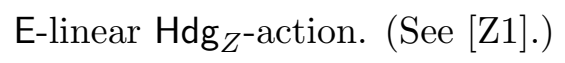

We now consider the case where the complex K3 surface $Z$ is the base-change from a quasi-canonical lift $\mathfrak{X}$ of an ordinary K3 surface $X$. 
Theorem 2.5. Assume $k$ is a finite field of $q$ elements and $X$ over $k$ is an ordinary $K 3$ surface. Let $L$ be a finite extension of $K$ with ring of integers $R$. Let $\mathfrak{X}$ be a quasi-canonical lift of $X$ and $X^{\circ}=\mathfrak{X} \otimes_{R}$ L. Let $\operatorname{Hdg}_{X}$ be the Hodge group of $\mathrm{M}\left(X_{\mathbb{C}}^{\circ}\right)$ and $\mathrm{E}=\operatorname{End}_{\operatorname{Hdg}_{X}} \mathrm{M}\left(X_{\mathbb{C}}^{\circ}\right)$ as above; $\mathrm{G}$ the Zariski closure over $\mathbb{Q}$ of the cyclic group generated by $\pi / q$ in $\mathrm{GL}\left(\mathrm{M}\left(X_{\mathbb{C}}^{\circ}\right)\right.$ ). Then we have $\mathrm{E}=\mathbb{Q}[\pi]$ and $\mathrm{Hdg}_{X}=\mathrm{G}$.

Proof. Since $\pi$ respects the Hodge structure on $\mathrm{M}\left(X_{\mathbb{C}}^{\circ}\right)$, we have $\pi \in \mathrm{E}$. Since the linear map $\pi$ is irreducible on $\mathrm{M}\left(X_{\mathbb{C}}^{\circ}\right)([\mathrm{Z} 2$, Theorem 1.1]), we have $\mathbb{Q}[\pi]=\mathrm{E}$ as $\mathrm{M}\left(X_{\mathbb{C}}^{\circ}\right)$ is an E-vector space.

Let $2 n=\operatorname{dim}_{\mathbb{Q}} \mathrm{E}$. Given an embedding $\mathrm{E} \subset \mathbb{C}$ sending $\pi$ to $\alpha \in \mathbb{C}$, it induces a character of the torus $\mathrm{G}$ by sending the generator $\pi / q$ to $\alpha / q$. In this way, we get $n$ independent characters ([Z2, Theorem 1.2]). Thus $\operatorname{dim}_{\mathbb{Q}} \mathrm{G} \geq \mathrm{n}$. On the other hand, every element in $G(\mathbb{Q})$ has norm one with respect to any embedding $\mathrm{E} \subset \mathbb{C}$. Thus $\operatorname{dim}_{\mathbb{Q}} \mathrm{G} \leq n$. Therefore $\operatorname{dim}_{\mathbb{Q}} \mathrm{G}=n$ and it is the maximal compact subtorus of $\operatorname{Res}_{\mathrm{E} / \mathbb{Q}} \mathbb{G}_{m}$. Consequently, $\mathrm{Hdg}=\mathrm{G}$ by the description of the shape of $\mathrm{Hdg}_{\mathrm{X}}$ and a dimension counting ([Z1, Remark 2.3.2]).

\section{Applications}

\section{A Torelli theorem}

3.1. Let $X$ be an ordinary $\mathrm{K} 3$ surface over a finite field $k$. Let $\mathfrak{X}$ over $W$ be the canonical lift of $X$. Write $X^{\circ}=\mathfrak{X}_{K}$. Let $T(X)=H^{2}\left(X^{\circ}(\mathbb{C}), \mathbb{Z}\right)$ and $V(X)=T(X) \otimes_{\mathbb{Z}} \mathbb{Q}=H^{2}\left(X^{\circ}(\mathbb{C}), \mathbb{Q}\right)$. The $\mathbb{Z}$-module $T(X)$ is equipped with a quadratic form induced from the cup product pairing. For any rational prime $\ell$, let $T_{\ell}(X)=T(X) \otimes_{\mathbb{Z}} \mathbb{Z}_{\ell}$ and $V_{\ell}(X)=V(X) \otimes_{\mathbb{Q}} \mathbb{Q}_{\ell}$. Then Lemma 2.3 says that the Frobenius morphism on $X$ over $k$ can be lifted as an endomorphism $\pi$ on $V(X)$.

Proposition 3.2. Assume $p>3$. The lifted Frobenius $\pi$ on $V(X)$ preserves the $\mathbb{Z}$-lattice $T(X)$.

Proof. We show that for any prime $\ell$, the lifted Frobenius $\pi$ preserves the $\mathbb{Z}_{\ell}$-lattice 
$T_{\ell}(X)$ in $V_{\ell}(X)$. For $\ell \neq p$, the map $\pi \otimes_{\mathbb{Q}} i d$ on $H^{2}\left(X^{\circ}(\mathbb{C}), \mathbb{Q}_{\ell}\right)$ is the geometric Frobenius on $H_{e t}^{2}\left(X_{\bar{k}}, \mathbb{Q}_{\ell}\right)$. Thus it preserves the lattice $H_{e t}^{2}\left(X_{\bar{k}}, \mathbb{Z}_{\ell}\right)=T_{\ell}(X)$.

At the prime $p$, since $\mathfrak{X}$ over $W$ is the canonical lift of $X$, by Lemma 1.6 , the Hodge filtration on $H_{d R}^{2}(\mathfrak{X} / W)$ coincides with the weight filtration on $H_{\text {cris }}^{2}(X / W)$. Thus the geometric Frobenius is an endomorphism on the filtered $\phi$-module $H_{c r i s}^{2}(X / W)$. Therefore $\pi$ preserves the $\mathbb{Z}_{p}$-lattice $H_{e t}^{2}\left(X_{\bar{K}}^{\circ}, \mathbb{Z}_{p}\right)=T_{p}(X)$ in $H_{e t}^{2}\left(X_{\bar{K}}^{\circ}, \mathbb{Q}_{p}\right)([\mathrm{FM}$, Remark 6.4]).

Theorem 3.3. (Cf. [N2, Theorem 2.1].) Assume $p>3$. Let $(X, I)$ and $(Y, J)$ be polarized ordinary $K 3$ surfaces over a finite field $k$ and $\left(T(X), \pi_{X}\right)$ and $\left(T(Y), \pi_{Y}\right)$ be the associated quadratic $\mathbb{Z}$-modules with lifted Frobenius endomorphisms of $X$ and $Y$, respectively. Let $\xi \in T(X)$ and $\eta \in T(Y)$ be the (twisted) first chern classes of $I$ and $J$, respectively. Suppose there exists an isometry

$$
f^{*}: T(Y) \rightarrow T(X)
$$

which commutes with the Frobenius and sends $\eta$ to $\xi$. Then there exists an isomorphism $f:(X, I) \rightarrow(Y, J)$ over $k$ such that $f_{\ell}^{*}:=f^{*} \otimes i d: T_{\ell}(Y) \rightarrow$ $T_{\ell}(X)$ are induced from $f$ on the étale cohomology under $T_{\ell}(X)=H_{e t}^{2}\left(X_{\mathbb{C}}^{\circ}, \mathbb{Z}_{\ell}\right)=$ $H_{e t}^{2}\left(X_{\bar{k}}, \mathbb{Z}_{\ell}\right)$ for all primes $\ell \neq p$.

Proof. One follows the same line as in the proof of [N2, Theorem 2.1] and takes care of the field of definition.

Let $(\mathfrak{X}, \mathfrak{I})$ and $(\mathfrak{Y}, \mathfrak{J})$ be the canonical lifts of $(X, I)$ and $(Y, J)$ respectively (cf. Lemma 1.4; [N1, Proposition 1.8]). Since the map $f^{*}: T(Y) \rightarrow T(X)$ commutes with the Frobenius $\pi_{Y}$ and $\pi_{X}$, it respects the Hodge structures by Lemma 2.3. Thus by the classical Torelli theorem for K3 surfaces over $\mathbb{C}$, there exists an isomorphism $f_{\mathbb{C}}:(\mathfrak{X}, \mathfrak{I})_{\mathbb{C}} \rightarrow(\mathfrak{Y}, \mathfrak{J})_{\mathbb{C}}$ that induces $f^{*}$. Choose an isomorphism $\bar{K} \simeq \mathbb{C}$, we may assume that the map $f_{\mathbb{C}}$ is defined over $\bar{K}$

$$
f_{\bar{K}}:(\mathfrak{X}, \mathfrak{I})_{\bar{K}} \rightarrow(\mathfrak{Y}, \mathfrak{J})_{\bar{K}} .
$$

Again, since the map $f_{\bar{K}}$ commutes with Frobenius, it is defined over a valuation field $L \subset \bar{K}$ with ring of integers $R$ such that the residue field of $R$ is $k$. Thus we get an isomorphism

$$
f_{L}:(\mathfrak{X}, \mathfrak{I})_{L} \rightarrow(\mathfrak{Y}, \mathfrak{J})_{L}
$$


such that $f_{\bar{K}}=f_{L} \otimes_{L} \bar{K}$.

Since the morphism $f_{L}$ is an isomorphism between the generic fibers of polarized smooth non-ruled schemes $(\mathfrak{X}, \mathfrak{I})$ and $(\mathfrak{Y}, \mathfrak{J})$ over the ring of integers $R$ of $L$, it can be extended uniquely to an isomorphism

$$
f_{R}:(\mathfrak{X}, \mathfrak{I})_{R} \rightarrow(\mathfrak{Y}, \mathfrak{J})_{R}
$$

In particular, we get the desired isomorphism

$$
f: X \rightarrow Y
$$

over $k$ by taking the reduction of $f_{R}$.

\section{The Hodge conjeture}

4.1. Let notations be as in $\S 2.1$ ( $k$ is finite). As in $\S 2.4$, we let $\mathrm{M}\left(X_{\mathbb{C}}^{\circ}\right)$ be the transcendental part of $H^{2}\left(X^{\circ}(\mathbb{C}), \mathbb{Q}(1)\right)$. The subspace $\mathrm{M}\left(X_{\mathbb{C}}^{\circ}\right)$ is self-dual via the induced cup product. Under this self-duality, we regard the lifted Frobenius $\pi$ on $\mathrm{M}\left(X_{\mathbb{C}}^{\circ}\right)$ as an element in $\mathrm{M}\left(X_{\mathbb{C}}^{\circ}\right)^{\otimes 2}$.

Proposition 4.2. With notations as above, the subspace of Hodge cycles in the tensor algebra of $\mathrm{M}\left(X_{\mathbb{C}}^{\circ}\right)$ is generated by the graphs $\Gamma_{n}$ of iterations $\pi^{n}$ of the lifted Frobenius $\pi$ in $\mathrm{M}\left(X_{\mathbb{C}}^{\circ}\right)^{\otimes 2}$.

Proof. Let $C^{n} \subset \mathrm{M}\left(X_{\mathbb{C}}^{\circ}\right)^{\otimes 2 n}$ be the subspace of Hodge cycles. Then by Theorem 2.5, the space $C^{n} \otimes_{\mathbb{Q}} \mathbb{Q}_{\ell} \subset \mathrm{M}\left(X_{\mathbb{C}}^{\circ}\right)^{\otimes 2 n} \otimes_{\mathbb{Q}} \mathbb{Q}_{\ell}$ coincides with Tate cycles in $\mathrm{M}\left(X_{\mathbb{C}}^{\circ}\right)^{\otimes 2 n} \otimes_{\mathbb{Q}} \mathbb{Q}_{\ell}$ regarded as a subspace in $H^{2}\left(X^{\circ}(\mathbb{C}), \mathbb{Q}_{\ell}(1)\right)^{\otimes 2 n}=$ $H_{e t}^{2}\left(X_{\bar{k}}, \mathbb{Q}_{\ell}(1)\right)^{\otimes 2 n}$. Since the $\mathbb{Q}_{\ell}$-space of Tate cycles in the tensor algebra of $\mathrm{M}\left(X_{\mathbb{C}}^{\circ}\right) \otimes_{\mathbb{Q}} \mathbb{Q}_{\ell}$, regarded as a subspace in $H_{e t}^{2}\left(X_{\bar{k}}, \mathbb{Q}_{\ell}(1)\right)$, is generated by $\Gamma_{n}$ ([Z3, Corollary 6.1.1]), the same holds true for the space of Hodge cycles in the tensor algebra of $\mathrm{M}\left(X_{\mathbb{C}}^{\circ}\right)$.

We remark that if the graphs $\Gamma_{n}$ are all algebraic, then Proposition 4.2 implies the validity of the Hodge conjecture for any self-product $X^{\circ} \times \cdots \times X^{\circ}$ of $X^{\circ}$ as $H^{2}\left(X^{\circ}(\mathbb{C}), \mathbb{Q}\right)=\mathrm{M}\left(X_{\mathbb{C}}^{\circ} \oplus \operatorname{NS}\left(X_{\mathbb{C}}^{\circ}\right)_{\mathbb{Q}}\right.$ and classes in $\mathrm{NS}\left(X_{\mathbb{C}}^{\circ}\right)_{\mathbb{Q}}, H^{0}\left(X^{\circ}(\mathbb{C}), \mathbb{Q}\right)$ and $H^{4}\left(X^{\circ}(\mathbb{C}), \mathbb{Q}\right)$ are all algebraic. 
We now focus on some examples.

A. Kummer surfaces.

4.3. Let $A$ be an ordinary abelian variety over a finite field $k$. Assume that the Frobenius morphism acts trivially on the 2-torsion points of $A$. Let $L$ be a finite extension of $K$ in $\bar{K}$ with ring of integers $R$. Let $\mathfrak{A}$ be a lift of $A$ over $R$ and $A^{\circ}$ be the generic fiber of $\mathfrak{A}$. Denote by $\delta: \widetilde{\mathfrak{A}} \rightarrow \mathfrak{A}$ the blow-up of $\mathfrak{A}$ along the 2 -torsion points of $\mathfrak{A}$. We have

$$
\delta^{*}: H^{2}\left(A^{\circ}(\mathbb{C}), \mathbb{Z}\right) \rightarrow H^{2}\left(\widetilde{A}^{\circ}(\mathbb{C}), \mathbb{Z}\right) \simeq H^{2}\left(A^{\circ}(\mathbb{C}), \mathbb{Z}\right) \oplus \mathbb{Z}(-1)^{\oplus 16},
$$

which, by extending the scalars, induces a homomorphism $\delta_{\ell}^{*}$ of $\operatorname{Gal}(\bar{K} / L)$ modules

$$
\delta_{\ell}^{*}: H_{e t}^{2}\left(A_{\bar{K}}, \mathbb{Z}_{\ell}\right) \rightarrow H_{e t}^{2}\left(\widetilde{A}_{\bar{K}}, \mathbb{Z}_{\ell}\right) \simeq H_{e t}^{2}\left(A_{\bar{K}}, \mathbb{Z}_{\ell}\right) \oplus \mathbb{Z}_{\ell}(-1)^{\oplus 16}
$$

for every prime number $\ell$.

Let $\mathfrak{X}$ be the Kummer surface associated with $\mathfrak{A}$ and $\epsilon: \widetilde{\mathfrak{A}} \rightarrow \mathfrak{X}$ the quotient map. Denote by $X$ and $X^{\circ}$ the special fiber and the generic fiber of $\mathfrak{X}$, respectively. In this case

$$
\epsilon^{*}: H^{2}\left(X^{\circ}, \mathbb{Q}\right) \rightarrow H^{2}\left(\widetilde{A}^{\circ}, \mathbb{Q}\right)
$$

is an isomorphism of rational Hodge structures and it induces an isomorphism $\epsilon_{\ell}^{*}$ of $\operatorname{Gal}(\bar{K} / L)$-modules

$$
\epsilon_{\ell}^{*}: H_{e t}^{2}\left(X_{\bar{K}}, \mathbb{Q}_{\ell}\right) \rightarrow H_{e t}^{2}\left(\widetilde{A}_{\bar{K}}, \mathbb{Q}_{\ell}\right)
$$

for every prime number $\ell$.

Lemma 4.4. With notations as above, we have the following:

a) The Kummer surface $X$ is an ordinary K3 surface.

b) The formal scheme $\mathfrak{X}$ is a quasi-canonical lift of $X$ if $\mathfrak{A}$ is a quasi-canonical lift of $A$.

c) If $\mathfrak{X}$ is a quasi-canonical lift of $X$, then $\mathfrak{A}$ is a quasi-canonical lift of $A$. 
d) If $L=K$ and $\mathfrak{A}$ is the canonical lift of $A$, then $\mathfrak{X}$ is the canonical lift of $X$.

Proof. a) Take a prime $\ell \neq p$. We have an isomorphism of $\operatorname{Gal}(\bar{k} / k)$-modules

$$
\epsilon_{\ell}^{*}: H_{e t}^{2}\left(X_{\bar{k}}, \mathbb{Q}_{\ell}\right) \stackrel{\sim}{\rightarrow} H_{e t}^{2}\left(\widetilde{A}_{\bar{k}}, \mathbb{Q}_{\ell}\right) .
$$

Thus the characteristic polynomial $f(X ; x)$ of the geometric Frobenius on $H_{e t}^{2}\left(X_{\bar{k}}, \mathbb{Q}_{\ell}\right)$ equals that $f(\widetilde{A} ; x)$ on $H_{e t}^{2}\left(\widetilde{A}_{\bar{k}}, \mathbb{Q}_{\ell}\right)$. Since

$$
H_{e t}^{2}\left(\widetilde{A}_{\bar{k}}, \mathbb{Q}_{\ell}\right)=H_{e t}^{2}\left(A_{\bar{k}}, \mathbb{Q}_{\ell}\right) \oplus \mathbb{Q}_{\ell}(-1)^{\oplus 16}
$$

and $A$ is ordinary, there exists a unique root of $f(\widetilde{A} ; x)$ which is a $p$-adic unit. Therefore $X$ is ordinary.

b) If $\mathfrak{A}$ is a quasi-canonical lift of $A$, then

$$
H_{e t}^{1}\left(A_{\bar{K}}^{\circ}, \mathbb{Q}_{p}\right)=H_{0} \oplus H_{1}
$$

with

$$
H_{i} \otimes_{\mathbb{Q}_{p}} \mathbb{C}_{p} \simeq \mathbb{C}_{p}(-i)^{\oplus 2}
$$

as $\operatorname{Gal}(\bar{K} / L)$-modules. Thus

$$
H_{e t}^{2}\left(A_{\bar{K}}^{\circ}, \mathbb{Q}_{p}\right)=\left(H_{0} \wedge H_{0}\right) \oplus\left(H_{0} \otimes H_{1}\right) \oplus\left(H_{1} \wedge H_{1}\right)
$$

and therefore

$$
H_{e t}^{2}\left(X_{\bar{K}}^{\circ}, \mathbb{Q}_{p}\right)=V_{0} \oplus V_{1} \oplus V_{2},
$$

where

$$
\begin{aligned}
& V_{0}=\left(\epsilon_{p}^{*}\right)^{-1}\left(H_{0} \wedge H_{0}\right) \\
& V_{1}=\left(\epsilon_{p}^{*}\right)^{-1}\left(H_{0} \otimes H_{1} \oplus \mathbb{Q}_{p}(-1)^{\oplus 16}\right) \\
& V_{2}=\left(\epsilon_{p}^{*}\right)^{-1}\left(H_{1} \wedge H_{1}\right) .
\end{aligned}
$$

Since

$$
V_{i} \otimes \mathbb{C}_{p} \simeq \mathbb{C}_{p}(-i)^{\oplus h_{i}},
$$

$\mathfrak{X}$ is a quasi-canonical lift by Lemma 1.5 .

c) Assume that $\mathfrak{X}$ is a quasi-canonical lift. Let $\mathfrak{B}$ be the Kuga-Satake abelian scheme over the ring of integers $R^{\prime}$ of a finite extension $L^{\prime}$ of $L$ insider $\bar{K}$. Then 
after replacing $L^{\prime}$ by another finite extension, $\mathfrak{A} \otimes_{R} R^{\prime}$ is isogenous to a factor of $\mathfrak{B}$ ([Sk, Theorem 2]). Since $\mathfrak{B}$ is a quasi-canonical lift of its special fiber (cf. [N1, Corollary 2.8]), we have

$$
H_{e t}^{1}\left(\mathfrak{B}_{\bar{K}}, \mathbb{Q}_{p}\right)=H_{0} \oplus H_{1}
$$

with

$$
H_{i} \otimes_{\mathbb{Q}_{p}} \mathbb{C}_{p} \simeq \mathbb{C}_{p}(-i)^{\oplus h} .
$$

Consequently $H_{e t}^{1}\left(A_{\bar{K}}^{\circ}, \mathbb{Q}_{p}\right)$ has a similar decomposition as a $\operatorname{Gal}\left(\bar{K} / L^{\prime}\right)$-module. Thus $\mathfrak{A} \otimes_{R} R^{\prime}$ is a quasi-canonical lift of $A$. Since the deformation of $A$ is prorepresentable, it is left exact. Therefore $\mathfrak{A}$ is a quasi-canonical lift of $A$ to $R$.

d) As in c), since the deformation of $X$ is pro-representable, we may replace $k$ by its algebraic closure. Thus we assume that $k$ is algebraically closed. Since $\mathfrak{X}$ is defined over $W$ and is a quasi-canonical lift by part b), it is the canonical lift of $X$ as there is only one torsion element in the (multiplicative) group $1+p W$ of principal units in $W$.

Theorem 4.5. Let $A$ be a complex abelian surface of CM type and $X$ the associated Kummer surface. Then the Hodge conjecture is true for any self product $X \times \cdots \times X$ of $X$.

Proof. If $A$ is isogenous to a product of two elliptic curves, Then the statement is true since the Hodge conjecture is known for products of arbitrary elliptic curves and the difference of $H^{2}(X, \mathbb{Q})$ and $H^{2}(A, \mathbb{Q}$ ) is algebraic (see $\S 4.3$ ). Henceforth, we assume that $A$ is simple. Since $A$ is of CM type, it is defined over a number field $F$. Write $E:=\left(\operatorname{End}_{F} A\right) \otimes_{\mathbb{Z}} \mathbb{Q}$, which is a CM field with $[E: \mathbb{Q}]=4$.

For a non-archimedean place $v$ of $F$, let $F_{v}$ be the completion of $F$ at $v$. Let $A_{v}=A \otimes_{F} F_{v}, \mathfrak{A}_{v}$ its Néron model and $\bar{A}_{v}$ the special fiber of $\mathfrak{A}_{v}$. Let $\pi_{v}$ be the Frobenius endomorphism of $\bar{A}_{v}$ relative to the residue field $k_{v}$ at $v$. After replacing $F$ by a finite extension, there exists a non-archimedean place $v$ such that the reduction $\bar{A}_{v}$ is a simple ordinary abelian variety over $k_{v}$ and the characteristic polynomial of $\pi_{v}$ has no multiple root ([Tan, Lemma 7.5]). Thus we have $\left(\operatorname{End}_{k_{v}} \bar{A}_{v}\right) \otimes_{\mathbb{Z}} \mathbb{Q}=\mathbb{Q}\left(\pi_{v}\right)$ and $\left[\mathbb{Q}\left(\pi_{v}\right): \mathbb{Q}\right]=4$ ([Tat, Theorem 2]). Consequently, as $\operatorname{End}_{F} A \rightarrow \operatorname{End}_{k_{v}} \bar{A}_{v}$ in injective, we have $E=\mathbb{Q}\left(\pi_{v}\right)$. 
Therefore $\pi_{v}$ can be lifted to an isogeny on $A_{v}$, which implies that $\mathfrak{A}_{v}$ is a quasicanonical lift of $\bar{A}_{v}$. Thus by Lemma $4.4, X$ can be regarded as the generic fiber of a quasi-canonical lift of the Kummer surface associated with $\bar{A}_{v}$ and by Proposition 4.2, the statement then follows.

B. Weighted K3 surfaces.

4.6. Let $X$ be one of the weighted $\mathrm{K} 3$ surfaces considered in [Yo] and [Yui]. There are two types of such an $X$ :

a). The K3 surface $X$ is the resolution of singularities of a certain hypersurface of Fermat type

$$
x_{0}^{n_{0}}+x_{1}^{n_{1}}+x_{2}^{n_{2}}+x_{3}^{n_{3}}
$$

in the weighted projective 3 -space $\mathbb{P}\left(m_{0}, m_{1}, m_{2}, m_{3}\right)$ (see [Yui, §2]). In this case, we consider $X$ as a surface defined over a number field $F$ containing $E:=\mathbb{Q}\left(\zeta_{n}\right)$ where $n$ is the least common multiple of $n_{i}, i=0, \ldots, 3$ and $\zeta_{n}$ is a primitive $n$-th root of unity. Then $E$ acts on any cohomology group of $X$ and this action commutes with the extra structure attached to that cohomology theory (Hodge structure, Galois action, ... ). There are fourteen K3 surfaces of this type.

b). The K3 surface $X$ is the resolution of singularities of a certain quasi-diagonal hypersurface

$$
x_{0}^{n_{0}}+x_{0} x_{1}^{n_{1}}+x_{2}^{n_{2}}+x_{3}^{n_{3}}
$$

in the weighted projective 3 -space $\mathbb{P}\left(m_{0}, m_{1}, m_{2}, m_{3}\right)$ (see [Yui, §3]). In this case, we consider $X$ as defined over a number field $F$ containing $E:=\mathbb{Q}\left(\zeta_{n}\right)$ where $n$ is the least common multiple of $n_{1}, n_{2}$, and $n_{3}$. Then $E$ acts on any cohomology group of $X$ and this action commutes with the extra structure attached to that cohomology theory. There are eighty five K3 surfaces of this type.

In both cases, we can find an open set $S$ of the ring of integers of $F$ such that $X$ can be extended to $\mathfrak{X}$ over $S$. For a finite place $v$ of $S$, let $F_{v}$ be the completion of $F$ at $v, R_{v}$ the ring of integers of $F_{v}$ and $k_{v}$ the residue field of $R_{v}$. Set $X_{v}=X \otimes_{F} F_{v}, \mathfrak{X}_{v}=\mathfrak{X} \otimes R_{v}$ and $\bar{X}_{v}$ the reduction of $\mathfrak{X}_{v}$. 
Theorem 4.7. Let $X$ be as above. Then there exists a finite place $v$ of $F$ such that $\bar{X}_{v}$ is an ordinary $K 3$ surface over the finite field $k_{v}$, and $\mathfrak{X}_{v}$ is a quasi-canonical lift of $\bar{X}_{v}$. Consequently the Hodge conjecture is true for any self product $X \times \cdots \times X$ of $X$.

Proof. In fact, there exists a positive integer $m$ such that if $v$ is a place over a rational prime $p$ with $p \equiv 1(\bmod m)$, then the reduction $\bar{X}_{v}$ is ordinary ([GY, Proposition 3.8]). Moreover $\operatorname{rk} \operatorname{NS}\left(X_{\mathbb{C}}\right)=22-[E: \mathbb{Q}]=\operatorname{rkNS}\left(\bar{X}_{v} \otimes_{k_{v}} \bar{k}_{v}\right)([$ Yui, Proposition 6.2]). As in $\S 2.4$, we pick a prime $\ell \neq p$ and let $\mathrm{M}_{\ell}\left(\bar{X}_{v}\right)$ be the orthogonal complement of $\operatorname{NS}\left(\bar{X}_{v} \otimes_{k_{v}} \bar{k}_{v}\right)$ in $H_{e t}^{2}\left(\bar{X}_{v} \otimes_{k_{v}} \bar{k}_{v}, \mathbb{Q}_{\ell}(1)\right)$. Then both $\zeta_{n}$ and the Frobenius endomorphism $\pi$ on $\bar{X}_{v}$ can be regarded as algebraic cycles in $\mathrm{M}_{\ell}\left(\bar{X}_{v}\right)^{\otimes 2}$. Since $\operatorname{dim}_{\mathbb{Q}} \mathbb{Q}\left(\zeta_{n}\right)=\operatorname{dim}_{\mathbb{Q}_{\ell}} \mathrm{M}_{\ell}\left(\bar{X}_{v}\right)=\operatorname{dim}_{\mathbb{Q}} \mathbb{Q}(\pi)([\mathrm{Z} 2$, Theorem 1.1]), we must have $\mathbb{Q}\left(\zeta_{n}\right)=\mathbb{Q}(\pi)$ ([Z3, Corollary 6.1.1]). Thus $\pi$ can be lifted to $H^{2}(X(\mathbb{C}), \mathbb{Q})$ and $X_{v}$ is a quasi-canonical lift of $\bar{X}_{v}$ by Theorem 1.8.

Remark 4.8. After this paper has been written, the author learned that in [Ma], it has been shown that the Hodge conjecture holds for arbitrary self products of any complex projective K3 surface whose Hodge endomorphism algebra on its transcendental lattice is a CM field. In particular this covers our examples here. The proof in $[\mathrm{Ma}]$ relies on a result of Mukai on the study of moduli of projective bundles over complex K3 surfaces and isogenies between K3 surfaces, and is different from our reduction approach.

\section{References}

[AM] M. Artin and B. Mazur, Formal groups arising from algebraic varieties. Ann. Sci. de l'E.N.S., 4e série, 10 (1977), 87-131.

[BO] P. Berthelot and A. Ogus, F-isocrystals and de Rham cohomology. I. Invent. Math. 72 (1983), 159-199.

[D1] P. Deligne, La conjecture de Weil pour les surfaces K3. Invent. Math. 15 (1972), 206-226.

[D2] P. Deligne, Cristaux ordinaires et coordonnées canoniques. With the collaboration of L. Illusie. With an appendix by Nicholas M. Katz. Surfaces 
Algébriques. Séminaire de Géométrie Algébrique d’Orsay 1976-78, Lecture Notes in Math., 868, pp. 80-137, Springer, Berlin-New York, 1981.

[F] J.-M. Fontaine, Représentations p-adiques semi-stables. Périodes p-adiques (Bures-sur-Yvette, 1988), Astérisque 223 (1994), 321-347.

[FM] J.-M. Fontaine and W. Messing, $p$-adic periods and $p$-adic étale cohomology. Current trends in arithmetical algebraic geometry (Arcata, Calif., 1985), 179-207, Contemp. Math., 67, Amer. Math. Soc., Providence, RI, 1987.

[GY] F. Q. Gouvêa and N. Yui, Arithmetic of diagonal hypersurfaces over finite fields. London Mathematical Society Lecture Note Series 209. Cambridge University Press, Cambridge, 1995.

[LST] J. Lubin, J.-P. Serre and J. Tate, Elliptic curves and formal groups. Lecture notes prepared in connection with the seminars held at the Summer Institute on Algebraic Geometry, Whitney Estate, Woods Hole, Massachusetts, July 6 - July 31, 1964. (Available at http://www.ma.utexas.edu/users/voloch/lst.html).

[Ma] J. J. Ramón Marí, On the Hodge conjecture for products of certain surfaces. Collect. Math. 59 (2008), no. 1, 1-26.

[N1] N. O. Nygaard, The Tate conjecture for ordinary K3 surfaces over finite fields. Invent. Math. 74 (1983), 213-237.

[N2] N. O. Nygaard, The Torelli theorem for ordinary K3 surfaces over finite fields. Arithmetic and geometry, Vol. I, 267-276, Progr. Math., 35, Birkhäuser Boston, Boston, MA, 1983.

[NO] N. O. Nygaard and A. Ogus, Tate's conjecture for K3 surfaces of finite height. Ann. of Math. 122 (1985), 461-507.

[Sk] A. N. Skorobogatov, The Kuga-Satake variety of a Kummer surface. Russian Math. Surveys 40, no.1 (1985), 243-244.

[Tan] S. G. Tankeev, On the weights of an $l$-adic representation and the arithmetic of Frobenius eigenvalues. Izv. Math. 63 (1999), 181-218. 
[Tat] J. Tate, Endomorphisms of abelian varieties over finite fields. Invent. Math. 2 (1966), 134-144.

[Yo] T. Yonemura, Hypersurface simple K3 singularities. Tohoku Math. J. 42 (1990), 351-380.

[Yui] N. Yui, Formal Brauer groups arising from certain weighted K3 surfaces. J. Pure Appl. Algebra 142 (1999), 271-296.

[Z1] Y. G. Zarhin, Hodge groups of K3 surfaces. J. Reine Angew. Math. 341 (1983), 193-220.

[Z2] Y. G. Zarhin, Transcendental cycles on ordinary K3 surfaces over finite fields. Duke Math. J. 72 (1993), 65-83.

[Z3] Y. G. Zarhin, The Tate conjecture for powers of ordinary K3 surfaces over finite fields. J. Algebraic Geom. 5 (1996), 151-172.

Jeng-Daw Yu

Department of Mathematics

National Taiwan University

Taipei, Taiwan

Email: jdyu@math.ntu.edu.tw 